he typical assessment of mediation compliance by physicians is similar to the assessment of an iceberg from the ship captain's window. The difference is that when a captain sees ice in the water, he assumes that what he sees might be only the "tip of the iceberg" requiring attention. In contrast, patients who inadvertently omit many doses and doctors who attribute poor control to lack of drug efficacy may have no concept that the underlying problem is poor compliance with the prescribed regimen. If the captain fails to recognise an iceberg in advance, he knows that he must turn his immense vessel rapidly to avoid disaster. Failing to recognise inadequate compliance as the source of the patient's problem, the physician is unaware of the appropriate action to be taken. Instead, the physician typically prescribes even more medication as a higher dose, or an alternative or second drug. Unfortunately, the patient often remains on a potentially fatal collision course. Why is this scenario so common in medical practice?

Inherent in the answers to these questions is a message for every clinician who prescribes medications: Look under the surface. Don't assume that you know which patients take their medication regularly. Don't assume that failure to control hypertension, hyperlipidaemia or other measures of cardiovascular disease is caused by lack of efficacy of the prescribed medications.

In daily practice, after the physician determines the diagnosis and selects an appropriate treatment, the burden of achieving a good outcome is shifted to the patient. Depending on the setting, patients might be left to accomplish this important task of self management with little guidance. Both physicians and patients need to understand that key factors affecting outcome are "compliance" (that is, attempting to take the medication each day as prescribed) and "persistence" (that is, continuing to take the medication long term). ${ }^{1}$ We know that the current treatment style does not work well because national surveys continue to demonstrate that only $23 \%$ of people with diagnosed hypertension have blood pressure measurements within the target range. Why is effectiveness so low? The diagnoses and prescribed treatments probably are appropriate. The patients heard the diagnosis and received the prescriptions. Why were the prescriptions not filled, or refilled? Why were doses not taken daily or long term? Where is the weak link in the system? Compliance with medication regimens is the link between disease management and attainment of the desired treatment outcomes.

Drugs don't work for patients who don't take them

\title{
DEFINITIONS
}

Correspondence to: Joyce Cramer, Yale University School of Medicine, 950 Campbell Avenue (G7E), West Haven, CT 06516-2770, USA; joyce.cramer@yale.edu
Compliance in the medical setting can be defined as when a patient follows mutually agreeable instructions prescribed by a healthcare provider. Another aspect of compliance is treatment persistence, with a focus on long term continuation of treatment. Both definitions include the concept of partial compliance ranging from the occasional missed dose to the occasional extra dose. The pattern for partial compliance may be erratic, or it may be consistent but different from what the physician prescribed. Patients who are partial compliers are making an effort to participate in their treatment, but neither achieve their intention nor receive the full effect of their treatment. Common reasons are forgetfulness and feeling that treatment is not necessary. Persistence is an issue when patients feel that they no longer need medication. Some people test themselves by purposefully omitting doses, while others simply become lax about daily dosing. Medication compliance for symptomatic and asymptomatic disorders is highly variable among the adult population. Approximately three quarters of medication is taken as prescribed, ${ }^{2}$ across a wide range of medical disorders. Simple dose regimens are easier to follow. However, compliance rates are most highly correlated with the number of doses rather than the number of medications or tablets that must be taken daily. The core issue for patients is: "How many times a day must I remember to take a dose?" 


\section{MEASURING COMPLIANCE}

Physicians have been concerned about whether patients were following their medical instructions since the time of Hippocrates. Unfortunately, the simple method of asking patients whether they took their medication is not a very accurate measure of compliance. Patients who know they have missed doses tend to tell physicians what they think the physician wants to hear to avoid embarrassment. ${ }^{2}$ If forgetfulness about dosing has been the main problem, the patient might not realise the frequency of missed doses. Reporting good compliance is not deceit, but lack of awareness of the problem. Large medical centres have the capacity to assess compliance based on the frequency of prescription refills. Analyses of large databases can provide an estimate of overall compliance long term without the details of whether doses were omitted occasionally or whether the pattern included long periods with no doses.

The newest technology to assess compliance is continuous electronic monitoring ${ }^{3}$ (MEMS, Medication Event Monitoring Systems, AARDEX, Zug, Switzerland). These units use a standard prescription bottle that has a microprocessor in the cap to record the date and time whenever the bottle is opened. ${ }^{34}$ Data from the units can be downloaded to a computer for a visual representation of how often the patient took the medication, the number of hours between doses, and periods of missed dosing. Electronic monitoring has given us a window on patient behaviour and the opportunity to study the link between compliance and treatment outcome. Most patients take approximately $50-90 \%$ of doses, although the overall range is $0-100 \%$. On average, patients treated for a variety of medical disorders take approximately $75 \%$ of medication as prescribed, irrespective of the potential for negative consequences. ${ }^{25}$ Neuropsychological correlates showed that compliance does not correlate with intelligence, memory, personality disorder, age, or education. The number of drugs a patient takes also does not correlate with compliance. Patients who are prescribed several medications tend to take all types of drugs together (for example, three medications, six pills with breakfast) or forget all of them when they miss that dose. $^{2}$ The conclusion is that the number of medications is not as important as the number of times a day doses must be remembered.

Electronic monitoring has proven the widely help belief that compliance diminishes when the number of doses per day increases. An overview of 76 reports using electronic monitoring showed mean (SD) compliance rates of 79 (14)\% for once daily, 69 (15)\% for twice daily, 65 (16)\% for three times daily, and $51(20) \%$ for four times daily dosing $(p<0.001)$ for treatment of a variety of medical disorders. ${ }^{1}$. Precision of dosing was even lower with only $59 \%$ of doses taken at appropriate time intervals. A review of 13 studies showed that compliance rates for once daily antihypertensive medications ranged from $55-86 \%$, averaging $76 \%{ }^{5}$

\section{People take approximately $75 \%$ of doses as prescribed, across a variety of medical disorders}

\section{PATTERNS OF COMPLIANCE}

Cardiovascular disease is a chronic disorder, requiring long term treatment. Medication taking behaviour starts with the first prescription and continues for a lifetime. Prescriptions for antihypertensive and lipid lowering medications are often given to patients based on the assumption that people are reluctant to participate in lifestyle modification. Physicians seem to think that patients who will not diet and exercise to improve their health will be willing to take medication. The result is that even simple once daily medications are not taken regularly. When blood pressure and lipids have been maintained at target levels for a long time, patients might feel that the medication is no longer necessary. A study of elderly patients newly treated for hypertension revealed that they filled prescriptions covering only $49 \%$ of days during the first year. ${ }^{6}$ Studies of treatment persistence have shown that half of patients discontinue lipid lowering treatment within five years. ${ }^{7}$ However, patients with comorbid diagnoses of hypertension, diabetes or coronary artery disease had significantly better compliance rates than those with only hyperlipidaemia.

Patients who take medication can be categorised as near optimal compliers, partial compliers, or non-compliers. ${ }^{8}$ The proportion of patients who have been prescribed an antihypertensive or lipid lowering medication but do not fill or refill the prescription is unknown, but assumed to be large. Among compliers, dosing can vary from day to day, or month to month, resulting in periods without treatment. I found that compliance was significantly higher during five day periods before and after medical appointments, compared to 30 days after a visit," a phenomenon I call "white-coat compliance". These data suggest that attention to dosing was enhanced in anticipation of the visit when health behaviour was a prominent issue. This behaviour was maintained for at least a brief period after the visit. Fading of health behaviour was associated with erratic compliance within a few weeks and the potential for medical problems because of under dosing. Thus, blood pressure readings in the medical office are likely to be the result of recent dosing but might not reflect a steady state measurement. Similarly, lipid concentrations might reflect careful dieting for a few days before the blood test.

\section{Did the drug fail, or did the patient fail}

to take the drug?

\section{COMPLIANCE AND OUTCOMES}

The relation between compliance with lipid lowering drugs and cardiovascular risk was demonstrated in the Lipid Research Clinic's coronary primary prevention trial. ${ }^{10}$ The more doses taken, the lower the cardiovascular risk, with the greatest benefit achieved for patients who took full doses of cholestyramine daily. These data are included in the medication label to explain the value of full compliance. The Helsinki heart study demonstrated that lipid reductions were linearly related to gemfibrozil compliance. ${ }^{11}$ Effectiveness was greatly reduced among patients who took less than $70 \%$ of prescribed doses. Other studies have shown that patients readmitted to the hospital because of uncontrolled blood pressure had used significantly less medication than patients who were not readmitted $(26 \% \vee 9 \%$ of days without medications). ${ }^{12}$ A small study with electronic monitoring showed a significant correlation between ambulatory diastolic blood pressure and mean compliance. ${ }^{13}$

\section{CONSEQUENCES OF ERRATIC COMPLIANCE}

Stopping and restarting an antihypertensive medication can be dangerous. Studies have demonstrated that omission of dose of a short acting calcium channel blocker or $\beta$ blocker resulted in significantly increased systolic and diastolic pressure and heart rate during the following two days, with 


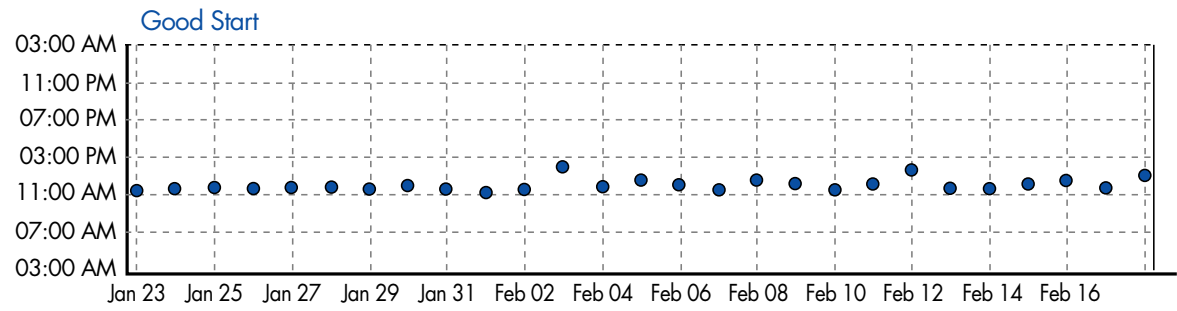

Figure 1 Example of initially good compliance, followed by erratic dose timing and many dose omissions (PowerView software, AARDEX Ltd, Zug, Switzerland).
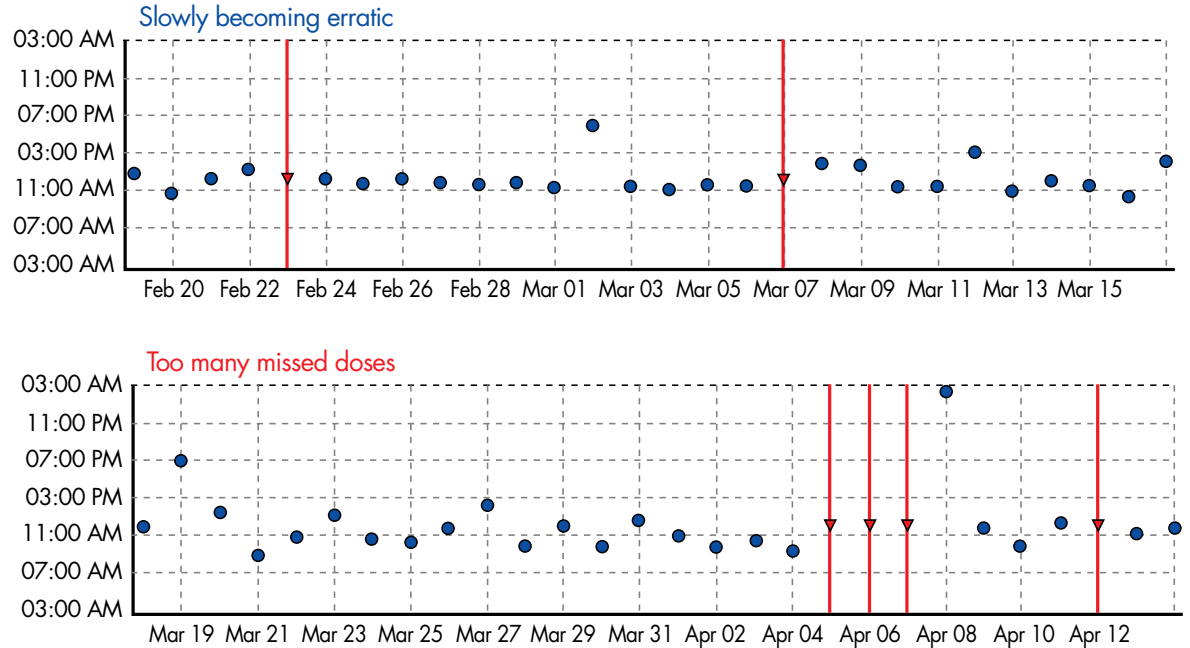

three patients developing rebound hypertension. ${ }^{14}$ Abrupt discontinuation of non-intrinsic sympathomimetic activity blockers can also result in rebound hypertension. ${ }^{15}$ Doxazosin, a peripheral vasodilator, can cause severe problems if doses are omitted for several days. The risk of a cardiovascular event was fourfold higher among patients who took less than $80 \%$ of their $\beta$ blocker medication, and twofold higher among those taking $80-90 \%$ of doses than patients who took more than $90 \%$ of doses. ${ }^{16}$ Note also that some cardiac antiarrhythmic drugs have a proarrhythmic effect when not taken as directed. This is an important lesson when prescribing medication that might do more transient harm than the overall beneficial effect of treatment for patients who are erratic compliers.

What can doctors do? Teach your

patients simple skills on how to follow a dosing plan, and reinforce the message at every visit

\section{STRATEGIES FOR IMPROVING MEDICATION COMPLIANCE}

Some of the essential aspects of prescribing that will enhance patient compliance are:
- selecting the fewest number of doses to be taken daily

- taking into consideration other medications the patient must take

- scheduling when doses are to be taken

- helping the patient select a reminder cue. ${ }^{17}$

A cue can be any activity that patients perform regularly that can be mentally associated with a scheduled dose. Basic cues are clock times, meal times, or daily rituals. For example, recommend that patients select specific clock times as dose times (for example, 7 am and $7 \mathrm{pm}$ ), or plan to take doses with meals (if they eat meals regularly). Other good cues are shaving, fixing one's hair, walking the dog, or listening to the news broadcast. Making the suggestion at the time the prescription is written will emphasise the importance of regular dosing, and takes minimal extra time. Everyone in the clinic or practice should reinforce the need for daily dosing with every patient, at every visit. Ask patients about their cue and how well it reminds them to take their medication. If the cue is not helping, suggest they choose another type of reminder. The message should stress the need for long term treatment to maintain persistence.

A combination of reminder cues and visual feedback of compliance data from electronic monitors is an effective method for improving compliance. I developed a Medication

Table 1 Electronic dosing record for a patient prescribed a medication to be taken three times a day. The calendar plot reveals erratic compliance on weekdays, and neglect on weekends

\begin{tabular}{lllllll}
\hline Sunday & Monday & Tuesday & Wednesday & Thursday & Friday & Saturday \\
\hline & 3 & 3 & 2 & 3 & 2 & 1 \\
1 & 3 & 3 & 3 & 2 & 2 & 0 \\
0 & 0 & 3 & 2 & 2 & 2 & 1 \\
0 & 2 & 2 & 2 & 2 & 1 & 0 \\
0 & 0 & 1 & 2 & 0 & & \\
\hline
\end{tabular}


Usage Skills for Effectiveness Program as a rapid, simple teaching programme that can be initiated by non-medical personnel spending a few minutes with the patient. ${ }^{18}$ The patients sees a record of all doses taken on a computer screen in a calendar format listing the number of doses taken each day, the dose times, or a figure showing dosing over several months. The report (fig 1 and table 1) is reviewed with the patient, asking about special problems on days when doses were missed, and how reminders can be used to improve compliance. This technique takes only minutes by any staff person to teach skills that might be useful for a lifetime.

Patients should develop a personalised plan to take their medication every day, 365 day a year, including holidays

\section{SUMMARY}

Physicians can picture themselves in the role of the ship's captain who sees ice in the water. Having learned about the high incidence of inadequate compliance and persistence, it is clear that every ice floe must be investigated to avoid potential disaster. We do not have the sonar system that helps the ship's captain scan under the water, but we can use other methods to avoid problems. We know that:

- taking three quarters of doses as prescribed leaves a wide window for potential cardiac disaster that is further increased when treatment is discontinued

- interruptions in the pharmacodynamic action of antihypertensive medications may compromise health in the short term or long term

- efficacy of some lipid lowering drugs is dose related.

The collision course includes target organ damage over time.

The American Heart Association has recommended use of strategies to enhance compliance to decrease morbidity and mortality from cardiovascular disease and stroke. ${ }^{19}$ The first task should be to help partial compliers develop better dosing habits to help to achieve this goal. Physicians who routinely discuss compliance and dose schedules, and who help patients select personalised cue reminders, can engage patients in their own care. $^{20}$ Attention to compliance is a simple way to demonstrate special attention to patient care as well as improve medical success.

\section{REFERENCES}

1 Claxton AJ, Cramer JA, Pierce C. Medication compliance: the importance of the dosing regimen. Clin Therapeutics 2001;23:1296-310.

- Overview of 76 studies with electronic compliance monitoring describes dose taking and dose timing deficits across a wide variety of medical disorders.

2 Cramer JA, Mattson RH, Prevey ML, et al. How often is medication taken as prescribed? A novel assessment technique. JAMA 1989;261:3273-7.

- The first report of variable compliance using electronic monitoring demonstrated that compliance was imperfect despite potentially disastrous consequences.
3 Cramer JA. Microelectronic systems for monitoring and enhancing patient compliance with medication regimens. Drugs 1995;49:321-7.

- Spending a few minutes to show patients how to tailor their medication regimens to fit into their schedules enhances compliance. Electronic monitoring systems allow clinicians to better understand patient dose taking behaviours, and to utilise those data to help patients develop schedules that meet individual lifestyles.

4 Cramer JA. Medication use by the elderly: enhancing patient compliance in the elderly: role of packaging aids and monitoring. Drugs \& Aging 1998:12:7-15.

5 Cramer JA. Consequences of intermittent treatment for hypertension: the case for medication compliance and persistence. Am J Managed Care 1999;4: 1563-8

6 Monane M, Bohn R, Gurwitz J, et al. Compliance with antihypertensive therapy among elderly medicaid enrollees: the roles of age, gender, and race. Am J Public Health 1996:86: 1805-8.

- Despite the efficacy of antihypertensive treatment, non-compliance may contribute to suboptimal cardiovascular outcomes.

7 Avorn J, Monette J, Lacour A, et al. Persistence of use of lipid-lowering medications: a cross-national study. JAMA 1998;279:1458-62.

- A comparison of US and Canadian populations for persistence with lipid lowering medications.

8 Rudd P. Compliance with antihypertensive therapy: a shifting paradigm. Cardiol Rev 1994;25:230-40.

9 Cramer JA, Scheyer RD, Mattson RH. Compliance declines between clinic visits. Arch Intern Med 1990;150: 1509-10.

10 Coronary Drug Project Research Group. Influence of adherence to treatment and response of cholesterol on mortality in the coronary drug project. N Engl J Med 1980;303:1038-41.

- Demonstration of the link between compliance and cardiovascular benefit from lipid lowering treatment.

11 Manninen V, Elo MO, Frick $\mathrm{H}$, et al. Lipid alterations and decline in the incidence of coronary heart disease in the Helsinki heart study. JAMA 1988;260:641-51.

12 Maronde RF, Chan LS, Larsen FJ, et al. Underutilization of antihypertensive drugs and associated hospitalization. Med Care 1989;27:1159-66.

13 Burnier M, Schneider MP, Chiolero A, et al. Electronic compliance monitoring in resistant hypertension: the basis for rational therapeutic decisions. J Hypertension 2001;19:335-41.

- A demonstration of the usefulness of compliance intervention to bring previously uncontrolled hypertension under control.

14 Johnson BF, Whelton A. A study design for comparing the effects of missing daily doses of antihypertensive drugs. Am J Therapeutics 1994; 1:260-7.

- A controlled simulation of the effect of missing a few doses of antihypertensive medication.

15 Rangno RE, Langlois S. Comparison of withdrawal phenomena after propranolol, metroprolol, and pindolol. Br J Clin Pharmacol 1982:13(suppl 2):345S-51S.

16 Psaty BM, Koepsell TD, Wagner EH, et al. The relative risk of incident coronary heart disease associated with recently stopping use of beta blockers. JAMA 1990;263:1653-7.

17 Cramer JA. Overview of methods to measure and enhance patient compliance. In: Cramer JA, Spilker B, eds. Patient compliance in medical practice and clinical trials. New York: Raven Press, 1991.

18 Cramer JA, Rosenheck R. Enhancing medication compliance for people with serious mental illness. J Nervous Mental Dis 1999;187:52-4.

- Patients randomised to a brief compliance feedback programme had significantly higher compliance rates than those assigned to usual care.

19 Miller NH, Hill M, Kottke T, et al. The multilevel compliance challenge. Recommendations for a call to action: a statement for healthcare professionals. Circulation 1997;95:1085-90.

20 Waeber B, Burnier M, Brunner HR. How to improve adherence with prescribed treatment in hypertensive patients? J Cardiovasc Pharmacol 2000;35(suppl 3):S23-6.

- Various strategies to improve compliance concludes that the "motivation of the patient to follow the treatment requires the doctor to be equally motivated". 